\title{
Biosimilaires : de la technique au médicoéconomique
}

\author{
Danièle Girault ${ }^{1}$, Jean-Hugues Trouvin ${ }^{2}$, Corinne Blachier-Poisson ${ }^{3}$, François Gary ${ }^{4}$, Didier Laloye ${ }^{5}$, et les
} participants à la table ronde $N^{\circ} 3$ de Giens XXX : Jean-François Bergmann ${ }^{6}$, Nicole Casadevall ${ }^{7}$, Cécile Delval ${ }^{8}$, Rima De Sahb Berkovitch ${ }^{9}$, Jean-Yves Fagon ${ }^{10}$, Marta Gersberg ${ }^{11}$, Catherine Lassale ${ }^{12}$, Philippe Lechat ${ }^{13}$, Claire Le Jeunne ${ }^{14}$, Jean-Louis Montastruc ${ }^{15}$, Jean-Louis Prugnaud ${ }^{7}$, Christelle Ratignier-Carbonneil ${ }^{16}$ et Cécile Rey-Coquais ${ }^{17 \dagger}$

1 Laboratoire Novartis Pharma, Rueil Malmaison, France

2 Université Paris Descartes, Faculté de Pharmacie, Paris, France

3 Laboratoire Amgen, Neuilly-sur-Seine, France

4 Laboratoire Sandoz, Levallois Perret, France

5 Hospira France, Meudon la Forêt, France

6 Hôpital Lariboisière, AP-HP, Paris, France

7 Hôpital Saint Antoine, AP HP, Paris, France

8 ANSM, Saint Denis, France

9 Laboratoire MSD, Courbevoie, France

10 CEPS, HEGP, Paris, France

11 Marta Gersberg Conseil, Chatou, France

12 LEEM, Paris, France

13 Hôpital Saint Louis, AP-HP, Paris, France

14 Hôpital Cochin, AP-HP, Paris, France

15 CHU, Faculté de Médecine, Toulouse, France

16 CNAMTS, Paris, France

17 Laboratoire Pfizer, Paris, France

Texte reçu le 24 novembre 2014 ; accepté le 15 décembre 2014

\author{
Mots clés : \\ biosimilaire ; \\ information ; \\ accès au marché ; \\ traçabilité ; \\ plan de gestion \\ des risques ; \\ substitution ; \\ taux de pénétration \\ biosimilaire
}

\begin{abstract}
Résumé - Le médicament biosimilaire (BS) correspond à un médicament biologique déclaré comme similaire à un médicament biologique de référence et dont le plan de développement inclut des études de comparaison par rapport à la référence, afin de confirmer la similarité du produit au niveau qualité, sécurité préclinique, et profil d'efficacité et de sécurité cliniques dont l'immunogénicité. Les biosimilaires (BS) sont différents des génériques, tant par leur complexité moléculaire que par leur réglementation. Ils représentent des enjeux économiques considérables avec l'expiration dans les 5 prochaines années des brevets de nombreux biomédicaments couvrant des aires thérapeutiques majeures comme la cancérologie, la rhumatologie ou la gastroentérologie, qui représentent des dépenses importantes pour l'Assurance maladie. La table ronde (TR) a émis des recommandations pour que l'arrivée des BS soit un succès, en s'appuyant sur le médecin prescripteur comme acteur de l'information et de la confiance, en mettant en place un suivi des patients traités par biomédicaments, et en accélérant l'accès au marché de leurs biosimilaires.
\end{abstract}

Abréviations : voir en fin d'article.

\footnotetext{
$\dagger$ Les articles, analyses et propositions issus des Ateliers de Giens n’engagent que leurs auteurs et ne préjugent pas de la position de leur organisme de tutelle.
} 


\section{Introduction}

Les biosimilaires sont devenus un sujet d'actualité et d'intérêt général en septembre 2013 avec un article du projet de loi de financement de la Sécurité Sociale (PLFSS) 2014 introduisant le principe de substitution par le pharmacien. Alors que cette classe de médicaments poursuivait une vie tranquille, les tribunes institutionnelles, les représentants industriels, les universitaires et les media se sont emparés du sujet combinant les questions scientifiques et de maîtrise des dépenses de santé. Dans ce contexte polémique, la table ronde (TR) de Giens a abordé à la fois les aspects scientifiques et économiques du sujet en écoutant tous les points de vue afin de proposer une série de recommandations équilibrées pour aider à l'arrivée des biosimilaires (BS) dans les meilleures conditions. Dans une première partie, nous décrivons le concept réglementaire de biosimilaire, préalable à une bonne compréhension du sujet : cette définition a été proposée pour établir une distinction claire entre les « copies » des médicaments chimiques (génériques) et les « copies » des médicaments biologiques (biosimilaires). Cette distinction a des conséquences en termes de développement des biosimilaires que nous détaillerons dans un second temps. Nous pourrons alors aborder les aspects économiques : les enjeux financiers généraux, la problématique d'accès au marché en France et les conditions d'un développement réussi des biosimilaires en tirant les leçons de l'expérience générique. Nous pourrons alors conclure par nos 5 recommandations.

\section{Les définitions, origine du concept de médicaments biosimilaires}

Le médicament biosimilaire (BS) correspond à un médicament biologique déclaré comme similaire par rapport à un médicament biologique dit «de référence » ${ }^{[1]}$ pour lequel il aura été prouvé, par un plan de développement adapté, que son profil qualité, sécurité et efficacité est «similaire » au profil du médicament de référence. Une « copie» de molécule biologique ne pouvant être strictement identique mais seulement « similaire» au produit de référence, pour des raisons de différence de source biologique et de procédé de fabrication, elle est qualifiée de «biosimilaires » par contraction du terme officiel donné dans l'article 10 de la directive 2001/83: $:^{[2]}$ " médicament biologique similaire à un médicament biologique de référence ».

La Commission Européenne et ses groupes d'experts ont proposé, avec le statut de «biosimilaire », le concept de comparabilité et une approche intermédiaire à celle du médicament générique : "lorsqu'un médicament biologique qui est similaire à un médicament biologique de référence ne remplit pas les conditions figurant dans la définition des médicaments génériques, en raison notamment de différences liées à la matière première ou de différences entre les procédés de fabrication du médicament biologique et du médicament biologique de référence, les résultats des essais précliniques ou cliniques appropriés relatifs à ces conditions doivent être fournis ». ${ }^{[2]}$ Cette formulation réglementaire fait bien le distinguo entre un médicament générique et un médicament biosimilaire et stipule que le plan de développement d'un médicament biologique similaire devra être plus étayé que le développement d'un médicament générique (lequel est essentiellement fondé sur l'identité de la molécule chimique et les preuves de bioéquivalence), notamment pour tenir compte de la variabilité intrinsèque de ces molécules complexes ( $c f$. infra).

Ainsi, cette définition de médicament biosimilaire permet d'établir une distinction claire entre les copies des médicaments chimiques et celles des médicaments biologiques et d'en tirer des conséquences en termes de gestion de la traçabilité, de l'information des patients et prescripteurs ainsi que des pratiques de substitution ou d'interchangeabilité, ainsi que cela a été discuté par la TR de Giens.

Comme le précise l'Agence Européenne du Médicament (EMA), «Un médicament biosimilaire est un médicament biologique qui est développé pour être similaire à un médicament biologique existant (le "médicament de référence »). Les biosimilaires sont à distinguer des génériques, qui ont des structures chimiques plus simples et sont considérés comme identiques à leur médicament de référence $\gg .{ }^{[3]}$

Pour comprendre la dénomination spécifique de «biosimilaire », proposée pour les substances actives d'origine biologique, il est nécessaire de rappeler d'une part la définition d'un médicament biologique et d'autre part quelques caractéristiques physicochimiques, biologiques et structurelles de ces molécules qui les rendent plus difficilement « copiables ».

\subsection{Définition d'un produit biologique}

Le médicament biologique est défini par la directive 2001/83 ${ }^{[1]}$ dans son annexe 1, partie I : «Le médicament biologique est un produit dont la substance active est une substance biologique. Une substance biologique est une substance qui est produite à partir d'une source biologique ou qui en est extraite, et dont la caractérisation et la détermination de la qualité [profil qualité, y inclus les impuretés, les variants, les produits de dégradation, etc.] nécessitent une combinaison d'essais physico-chimiques et biologiques ainsi que la connaissance de son procédé de fabrication et de son contrôle ».... Cette définition confirme le fameux dogme selon lequel une substance biologique est en partie décrite par son procédé d'obtention ou encore que le «procédé fait le produit». 
La classification comme médicament biologique entraîne au plan réglementaire l'application de critères d'évaluation spécifiques du profil qualité, compte tenu de la complexité moléculaire des substances et des procédés d'obtention. Avec les produits biologiques, la qualité finale du médicament et sa reproductibilité inter-lots sont plus difficiles à maîtriser et à démontrer qu'avec les médicaments chimiques.

\subsection{Les spécificités moléculaires en biologie}

Les substances actives d'origine biologique présentent une série de caractéristiques tant au plan moléculaire que leur procédé d'obtention qui les distinguent des substances actives d'origine chimique. ${ }^{[4]}$ En premier lieu, ce sont des macromolécules de masse moléculaire allant de plusieurs dizaines à la centaine de kilodaltons - à comparer à la masse moléculaire de l'aspirine de 180 daltons - soit des structures moléculaires de 20 à 800 fois plus complexes, non accessibles par chimie de synthèse et nécessitant le recours à une source biologique. Ces macromolécules sont essentiellement représentées par la classe des protéines (hormones, cytokines, facteurs de croissance, anticorps monoclonaux) avec quelques représentants de glycanes complexes (héparine, héparines de bas poids moléculaire [HBPM]). Elles ont une organisation spatiale en trois dimensions qui doit être conservée pour le maintien de l'activité biologique, alors que les molécules d'origine chimique sont de structure plane avec une flexibilité très réduite et contrainte.

En sus de la structure moléculaire initiale, et notamment pour les protéines, il faut mentionner des attributs complémentaires (phénomènes de glycosylation, sulfatation, amidation, ponts disulfures, etc.) qui complexifient encore la structure finale des substances biologiques d'intérêt. Ces modifications de structure interviennent au cours du processus de biosynthèse et vont conduire à une diversité/variabilité des molécules produites décrite sous le terme de «micro hétérogénéité ». En effet, au sein d'un lot de production, on retrouve des « isoformes » et des «variants », dont la répartition qualitative et quantitative sera naturellement variable, avec des limites de variabilité encadrées et contrôlées par la maîtrise des procédés. Ainsi, le concept de «molécule unique », appliqué aux substances chimiques, ne peut pas s'appliquer aux substances biologiques, et il faut plutôt évoquer une «population moléculaire » de la molécule d'intérêt. Une grande partie des enjeux et défis des médicaments biologiques réside ainsi dans la mâ̂trise du profil moléculaire, de sa variabilité intrinsèque et de sa reproductibilité à chaque campagne de production. Cette constance dans le profil de qualité est importante car elle conditionne deux paramètres importants du profil clinique :

- Le profil d'efficacité et la réponse thérapeutique, dépendantes du respect de la structure tridimensionnelle de la molécule, du respect de son profil de glycosylation le cas échéant et globalement du maintien de ses attributs qualité. Ainsi, un profil de glycosylation différent ou une protéine mal glycosylée ou partiellement dégradée lors de la purification ou de la mise en forme pharmaceutique peut présenter une moindre efficacité qui pourrait ne pas être détectée par les méthodes analytiques appliquées lors des contrôles sur chaque lot de médicament.

- Le profil de sécurité, et surtout le risque de réaction immune : ces macromolécules sont des substances pouvant être immunogènes, ce d'autant que la molécule exogène diffère des motifs « du soi »... Le potentiel immunogène doit être vérifié lors de l'administration à l'Homme car toute réaction immune pourrait entraîner des effets indésirables allant de la perte d'activité (développement d'anticorps neutralisants) à des réactions d'intolérance (anaphylaxie, hypersensibilité notamment), même si l'apparition d'anticorps n'entraîne pas systématiquement une perte d'activité ou des réactions d'intolérance. A cette immunogénicité intrinsèque, le plus souvent déjà largement documentée par l'expérience clinique acquise avec le médicament de référence, s'ajoute le risque d'une « néo-antigénicité » provoquée par l'apparition de nouveaux motifs antigéniques sur la molécule, suite à une modification du profil moléculaire, le plus fréquemment due à une réaction de dégradation ou de mauvaise conservation du médicament. Ce risque ne peut pas toujours être détecté par les méthodes analytiques courantes mais sera en revanche détecté par l'organisme des patients traités, avec la possibilité d'induire une rupture de la tolérance immune, initialement développée vis à vis du médicament de qualité acceptable.

Par ailleurs, la source biologique d'où sont produites ou extraites ces substances biologiques est elle-même cause de variabilité dans le profil de qualité. En effet, le développement d'un biosimilaire suppose de facto la mise au point d'un nouveau procédé de fabrication qui peut lui-même varier sensiblement du procédé utilisé par le fabricant du médicament de référence : par exemple dans le cas des protéines recombinantes, le système d'expression et notamment la lignée cellulaire génétiquement modifiée ou encore les conditions opératoires et milieux de culture ou temps de contact. Peuvent aussi être modifiées les conditions d'extraction ou de purification fixées par le nouveau producteur en fonction de son expérience et de sa maîtrise dans le génie des procédés biologiques.

Comme précisé plus haut, ces différences dans le procédé de production peuvent éventuellement avoir des conséquences notables sur le profil qualité, qu'il faudra valider en terme d'impact sur les profils d'efficacité et de tolérance.

Un dernier élément à prendre en compte est celui de la difficulté à caractériser, analyser et contrôler les nombreux paramètres de qualité qui décrivent et vérifient, pour chaque lot de 
production, le profil de la «population moléculaire» décrite cidessus. Le contrôle avant libération de chaque lot de médicament destiné à l'usage clinique doit s'attacher à valider ces différents attributs qualité, qualitativement et quantitativement. Une seule méthode analytique est insuffisante à contrôler la totalité du profil et il va falloir utiliser, comme le précise la définition européenne « une combinaison d'essais physico-chimiques et biologiques...», ce d'autant que malgré les progrès des méthodes d'analyses, certaines parties de la molécule restent inaccessibles à l'analyse des méthodes utilisées en routine et à la détection de petites différences qui auraient pu intervenir ponctuellement d'un lot de production à un autre notamment. Ces différents éléments conduisent à considérer que chaque lot présente une certaine «variabilité intrinsèque » qui ne peut être ni prédite ni vérifiée, et que seule la maîtrise du procédé permet de garantir que la variabilité inter-lot n'excédera pas une certaine limite. Cette problématique d'analyse se retrouve également dans l'exercice de «comparaison» des profils de qualité du médicament de référence et du médicament biosimilaire ( $c f$. paragraphe 3 ).

En résumé, une substance biologique se distingue, par rapport à une substance d'origine chimique, par sa structure complexe et micro-hétérogène, son procédé de production et par la complexité des méthodes de contrôle à mettre en œuvre. Tous ces facteurs de complexité aboutissent à un niveau de « variabilité » entre les lots, qu'il faut pouvoir maîtriser afin de s'assurer de l'absence d'impact sur le profil d'efficacité et de sécurité d'emploi lorsque le médicament de référence ou son biosimilaire est administré au patient.

\section{Le développement d'un biosimilaire et les questions encore en suspens}

Le dossier de demande d'autorisation sur le marché (AMM) pour un médicament biosimilaire doit répondre à l'exigence réglementaire de l'article 10 de la directive ${ }^{[2]}$ selon lequel «... les résultats des essais précliniques ou cliniques appropriés relatifs à ces conditions doivent être fournis... »

La partie principale du développement concerne les aspects dits de «qualité pharmaceutique» par lesquels le développeur devra établir et décrire son procédé de production, de purification et la stratégie de contrôle qualité, afin de montrer qu'il est capable de produire de façon reproductible, et selon des normes de qualité acceptable, la molécule d'intérêt thérapeutique « copie». Celle-ci doit ensuite être comparée avec la molécule originale «de référence » afin de montrer que, dans ces nouvelles conditions de production, la molécule «copie » est similaire à la molécule de référence. Cet exercice de comparaison s'attachera à rechercher toutes les différences possibles sur tous les «attributs qualité » qui définissent le profil de pureté et d'activité biologique.
Cet exercice de «comparaison» doit faire appel à toute la batterie des méthodes analytiques évoquées précédemment, avec des méthodes analytiques spécifiques et sensibles pour estimer le niveau de «similarité » entre le biosimilaire et la molécule de référence. Les textes réglementaires ${ }^{[1,3,5,6]}$ précisent clairement qu'il n'est pas attendu que les attributs de qualité du biosimilaire soient identiques à ceux du médicament de référence. En effet, si le procédé de production fait appel à une source biologique différente (par exemple la cellule productrice est d'une lignée cellulaire d'une autre espèce animale), les impuretés issues du procédé seront différentes qualitativement et quantitativement. Cette situation peut être évoquée pour de nombreuses autres caractéristiques moléculaires tels que le profil de glycosylation ou le taux de variants peptidiques sur les extrémités $\mathrm{N}$ - et C-terminales, notamment pour les anticorps monoclonaux, comme le mentionne le rapport public d'évaluation des dossiers biosimilaires Inflectra ${ }^{\circledR} /$ Remsima $^{\circledR}$, copie de l'anticorps monoclonal infliximab (Remicade $\left.{ }^{\circledR}\right) .{ }^{[7]}$

Une fois les deux molécules princeps et biosimilaires comparées, et les différences moléculaires identifiées, le plan de développement non clinique et clinique du biosimilaire sera adapté en fonction de ces différences, afin de démontrer qu'elles n'impactent pas le profil d'efficacité et de sécurité d'emploi. Les études non cliniques et cliniques seront essentiellement comparatives entre la molécule de référence et son biosimilaire, intégrant le suivi de paramètres pharmacodynamiques, pharmacocinétiques pertinents, tant dans des approches in vitro ou expérimentales animales que dans une ou des études cliniques de pharmacologie/pharmacocinétique, voire une étude clinique d'efficacité, portant sur « une population la plus sensible » capable de détecter des différences d'efficacité ou de tolérance sur un échantillon relativement restreint. Le degré d'exigence en termes d'études cliniques est variable selon les produits et selon le degré de similarité qui aura été établi entre le princeps et le biosimilaire.

Pour les médicaments de référence qui présentent plusieurs indications thérapeutiques dans leur AMM, la question se pose de la légitimité d'octroyer au biosimilaire l'ensemble des indications, alors que le développement clinique n'aura envisagé qu'une étude d'efficacité dans une seule des indications. Cette question a été largement discutée dans les différents guidelines et très récemment encore argumentée. ${ }^{[8]}$ Elle fait essentiellement débat lorsque les indications portent sur des systèmes physiologiques différents (rhumatologie, gastroentérologie, dermatologie par exemple) et que l'essai clinique d'équivalence a été mené sur une indication ne représentant qu'un des systèmes concernés. Il en est de même pour les développements dans des populations différentes comme les indications pédiatriques. L'exemple le plus récent est celui du biosimilaire de l'infliximab. ${ }^{[7]}$ 
Ainsi, le plan de développement d'un biosimilaire repose sur la notion d'études de comparaison, sur les trois critères de qualité, sécurité et efficacité, afin de confirmer que les différences observées au niveau de la substance active ou du produit fini n'auront pas d'impact sur le profil d'efficacité et de sécurité clinique, établi depuis de nombreuses années pour le médicament biologique de référence. Toutefois, s'il permet d'assurer la comparaison entre le médicament de référence et son biosimilaire, ce plan de développement ne permet pas actuellement d'évaluer les conséquences, notamment en termes d'immunogénicité, en cas de substitution et d'alternance de traitement chez un patient qui a été traité pendant une première période par le médicament de référence et qui viendrait à être changé de traitement (par exemple en cas de substitution lorsqu'un biosimilaire est disponible), voire à revenir au traitement initial quelques mois plus tard ou être traité par un nouveau biosimilaire du même médicament de référence (ces modifications de traitement pour un patient sont usuellement qualifiées de «switch»).

Ces risques d'immunogénicité croisée, induits par les pratiques de «switch» que pourrait permettre ou encourager l'approche par substitution, ne sont pas couverts actuellement par les plans de développement proposés dans les guidelines européens et suggèrent donc qu'une surveillance active soit proposée lors de l'arrivée sur le marché des biosimilaires, comme en témoigne le plan de gestion de risque prévu pour le biosimilaire de l'infliximab. ${ }^{[7]}$ Au regard du plan de gestion de risque, et au-delà du risque potentiel d'immunogénicité, qui est spécifique aux biomédicaments, il faut par ailleurs rappeler que les biosimilaires, à la différence des médicaments génériques, sont considérés comme des «nouveaux médicaments » et à ce titre, l'octroi de leur AMM est assujetti à la mise en place d'un plan de pharmacovigilance dédié, qui inclura notamment des obligations de traçabilité et de suivi post-commercialisation, pour combler les incertitudes identifiées au moment de l'évaluation.

L'ensemble de ces éléments scientifiques et règlementaires exigés pour le développement d'un biosimilaire montre bien que nous avons affaire ici à un nouveau type de «copies » très différenciées des génériques. Cette réglementation spécifique permet à la fois de développer des biosimilaires pour un coût bien moindre que celui de la molécule de référence (un nombre limité d'études non cliniques et un développement clinique réduit à des études de pharmacodynamie/pharmacocinétique et de phase 3 dans une indication dite «modèle») mais qui, du fait de ces essais non cliniques et cliniques complémentaires, représentent des coûts de développement nettement supérieurs à celui des génériques, sans occulter le coût de la mise au point d'un nouveau système de production d'une molécule biologique complexe. Pour donner un ordre de grandeur, on parle de 3 à $5 \mathrm{M} €$ pour un dossier d'AMM de générique et de 100 à $250 \mathrm{M} €$ pour un dossier de biosimilaire, à comparer au 1 Milliard $€$ pour le développement complet d'une nouvelle molécule. Dans le contexte actuel de pression sur les finances des systèmes de santé européens, on voit très vite l'intérêt de l'introduction de médicaments aux coûts de développement moindres et au rapport bénéfice/risque équivalent.

\section{Les biosimilaires : une opportunité économique}

Le système de santé français fait face au double défi d'une demande croissante des dépenses de santé supérieure au rythme de croissance du produit intérieur brut (PIB) et d'un déficit structurel sur les 30 dernières années. Dans ce contexte, toute technologie médicale qui apporte un bénéfice médical équivalent pour un moindre coût sera saluée par le payeur.

Or, le marché des médicaments biologiques innovants (que par convention nous désignons dans cet article par le terme de «biomédicaments ») représente aujourd'hui un enjeu majeur en France et en Europe. Sur les 10 premiers médicaments en chiffres d'affaire commercialisés en France, 8 à l'hôpital et 4 en ville sont des biomédicaments. Le chiffre d'affaire total des biomédicaments en France représente 7 Md $€$ en 2013 avec une croissance de 2,5\% versus 2012 alors que le marché total était en récession. ${ }^{[9]}$ Parallèlement le nombre de médicaments biologiques enregistrés augmente progressivement chaque année (173 en 2013 versus 115 en 2004) avec l'arrivée de molécules toujours plus innovantes pour la prise en charge de pathologies coûteuses (cancer, maladies auto-immunes, etc.).

L'arrivée des biosimilaires visant ces pathologies coûteuses a un double intérêt économique à court terme : elle élargit l'offre en proposant des médicaments similaires à un prix inférieur pour ce qui est des biologiques dispensés en pharmacie d'officine et elle stimule la compétition en faisant baisser les prix du princeps. À long terme, elle pousse les laboratoires d'innovation à poursuivre leurs recherches et à développer les innovations futures.

L'introduction des premiers biosimilaires en France date de 2007 et a déjà permis des économies. La pénétration des biosimilaires en volume est respectivement pour l'hormone de croissance de $35 \%$ au sein de la molécule de référence et $13 \%$ dans la classe complète; pour les facteurs de croissance granulocyte colony-stimulating factor (GCSF), de $75 \%$ au sein de la molécule de référence et $20 \%$ dans la classe; pour les érythropoïétines, de $36 \%$ au sein de la molécule de référence et $10 \%$ dans la classe. L'ensemble de ces chiffres montre une prise de marché non négligeable qui signe bien la confiance grandissante des professionnels de santé, médecins et pharmaciens, dans ce type de médicaments. Il est à noter qu'en France, aucune mesure incitative n'a été mise en place pour soutenir le développement de ces 
premiers biosimilaires, contrairement à d'autres pays Européens comme l'Allemagne.

Mais l'intérêt économique majeur des biosimilaires pour notre système de santé est à venir. En effet, les chutes de brevet des biomédicaments à chiffre d'affaires (CA) élevé sont attendues dans les 3 à 5 prochaines années. Il s'agit principalement des anticorps monoclonaux utilisés dans des domaines thérapeutiques variés tels que l'oncologie, l'hématologie, la rhumatologie ou la gastroentérologie et dont l'arrivée des médicaments de référence dans les dix dernières années a entraîné des changements majeurs dans la prise en charge des maladies concernées. L'évaluation des économies potentielles apportées par ces biosimilaires est complexe à faire car elle dépend des politiques de prix et d'incitation.

\section{L'accès au marché des biosimilaires : de nouvelles règles à inventer?}

Si les biosimilaires bénéficient de règles d'octroi d'AMM spécifiques fortement distinctes de celles des génériques, la question d'une procédure d'accès au marché spécifique reste ouverte. A ce jour ni la Haute Autorité de Santé (HAS), en charge de rendre un avis au Ministre sur le remboursement au travers des avis de la commission de transparence, ni le Comité Économique des Produits de Santé (CEPS) en charge de la politique du prix du médicament remboursable n'ont une doctrine spécifique sur les biosimilaires.

Actuellement, la Commission de la Transparence applique aux biosimilaires les mêmes règles d'évaluation qu'à toute nouvelle molécule. Alors que les génériques, lorsqu'ils ne sont pas différents du princeps, sont exemptés d'une évaluation par la Commission de la Transparence et passent directement de la case AMM à la case négociations de prix pour accélérer leur accès au marché, rien de tel n'existe ou n'est envisagé pour les BS. A ce jour, seuls 8 BS ont été évalués par la commission. Selon la doctrine en vigueur, ces médicaments ont été évalués en tant que nouveaux médicaments, dans le cadre de procédure simplifiée. L'évaluation par la commission de ces 8 cas s'est toujours soldée par la même conclusion : le service médical rendu (SMR) a été identique au biologique de référence et sans amélioration du service médical rendu (ASMR) versus ce médicament de référence, ce qui est à la fois cohérent (tous les BS sont traités de la même manière) et logique (il s'agit de «copies » d'un médicament de référence). L'arrivée croissante de BS et un meilleur recul sur les données au long cours de ces produits pourraient changer la position de la HAS et conduire au développement d'une procédure spécifique des BS encore plus simplifiée.

Du côté du CEPS, la doctrine reste à élaborer pour la ville. Concernant l'hôpital, les orientations actuelles consistent à baisser les prix du produit de référence de $10 \%$, d'aligner dans un premier temps le prix du BS avec le produit de référence, et de réaliser rapidement (un an plus tard) la révision du tarif du BS. La définition d'une doctrine définitive dépendra du traitement des BS dans les évaluations qui précèdent la fixation du prix, déterminantes pour le CEPS : politique de prix identique à celle de nouveaux produits obtenant une ASMR 5 ou à celle des génériques ou encore création d'une troisième catégorie bénéficiant de règles spécifiques?

Le modèle économique de développement des BS ne permet probablement pas un alignement avec les règles du générique (-60\% au lancement versus le princeps de référence). En revanche, pour donner plus de visibilité aux industriels, il apparait important de fournir des règles stables et transparentes qui permettront de mieux anticiper la viabilité d'un lancement de BS et sa pérennité sur le marché français. Un nouveau modèle de politique de prix (au lancement et dans la durée) est aussi à concevoir.

\section{Quelles conditions à une introduction réussie des biosimilaires en France?}

Pour répondre à cette question, il est intéressant d'analyser l'expérience passée des génériques et en tirer les réussites et les échecs.

Depuis leur introduction en France dans les années 1980, les médicaments génériques souffrent d'une absence de reconnaissance tant de la part du corps médical que des patients. La France est l'un des derniers pays dans le monde où des débats continuent à mettre en doute la qualité et parfois même l'efficacité des médicaments génériques. Encore aujourd'hui «la place des génériques reste notablement moins importante que chez nos voisins allemands ou anglais ». ${ }^{[10]}$

L'analyse a posteriori montre clairement que plusieurs éléments peuvent expliquer cette situation : une absence de communication scientifique et institutionnelle cohérente auprès des professionnels de santé et des patients pour rassurer sur la qualité de ces produits, un développement exclusivement sous l'angle économique, des messages grand public contradictoires, une appétence forte des médecins français pour les médicaments nouveaux ( l'effet de structure » souligné dans le rapport du CEPS 2013), des sociétés savantes très réservées, un périmètre des génériques circonscrit par une liste positive, une absence d'incitation positive pour les médecins, et un développement du marché des génériques confié en majorité aux pharmaciens d'officine par le droit de substitution, les avantages commerciaux, et les incitations à la substitution (tiers payant contre générique). Ainsi, un dispositif centré sur les incitations financières et une absence totale de communication institutionnelle pédagogique et positive expliquent le relatif échec de la politique des génériques. 
L'apparition des médicaments biosimilaires dans la politique de santé remonte à l'accord cadre Comité Economique des Produits de Santé/Les Entreprises du Médicament (CEPS/LEEM), signé le 5 décembre $2012^{[11]}$ qui précise que «les parties signataires conviennent de l'enjeu que représente le développement des médicaments biosimilaires pour contribuer au financement du progrès thérapeutique».

Le Conseil Stratégique des Industries de Santé (CSIS, 5 juillet 2013), relancé sous la présidence du Premier Ministre, a mis en perspective les attentes des différents ministères sur les médicaments biosimilaires (mesure 13 : biosimilaires) : ${ }^{[12]}$ «Assurer la sécurité des patients tout en permettant la réalisation d'économies par l'Assurance Maladie et le développement des biosimilaires en France ». Parmi les actions proposées figure «La mise en place d'un cadre spécifique aux biosimilaires qui permettra de faciliter la mise à disposition des biosimilaires par les industriels et le développement de leur utilisation par les prescripteurs et les patients ».

L'apparition dans la LFSS 2014 (Article 47) de la possibilité d'un droit de substitution par le pharmacien pour les médicaments biosimilaires en initiation de traitement, avant même que le groupe de travail du CSIS ne se mette en place, va accélérer la mobilisation de toutes les parties prenantes sur ce sujet. «Liste de référence des groupes biologiques similaires », «droit de substitution », autant de termes créant une confusion avec les génériques et faisant craindre les mêmes erreurs. ${ }^{[13]}$

En septembre 2013, l'Agence Nationale de Sécurité du Médicament (ANSM) publie un rapport sur les médicaments biosimilaires et préconise la recommandation suivante : «après une première administration, ne pas modifier le produit administré au patient afin de limiter les risques d'immunisation et d'assurer la traçabilité du suivi de pharmacovigilance ». ${ }^{[14]}$

Le groupe de travail CSIS Biosimilaires, réuni à partir de début 2014 et regroupant industriels, ministères de la santé et de l'industrie, agences de santé, Caisse Nationale d'Assurance Maladie (CNAM) et professionnels de santé, a permis de mettre en perspective les difficultés techniques et pratiques inhérentes à la mise en place du « décret substitution », mais a surtout renforcé l'idée que créer un environnement favorable à la prescription et à la délivrance des médicaments biosimilaires nécessitait une autre approche : s'appuyer en priorité sur le corps médical, afin de créer la confiance chez les patients. Introduire le droit de substitution dans la pharmacie d'officine dès 2014 était prématuré et pouvait avoir des effets contre-productifs.

Dans la TR de Giens comme dans le groupe de travail du CSIS, le consensus s'est unanimement fait autour du médecin acteur de l'information et de la confiance, aussi bien en initiation de traitement que lors des changements éventuels pendant le traitement.

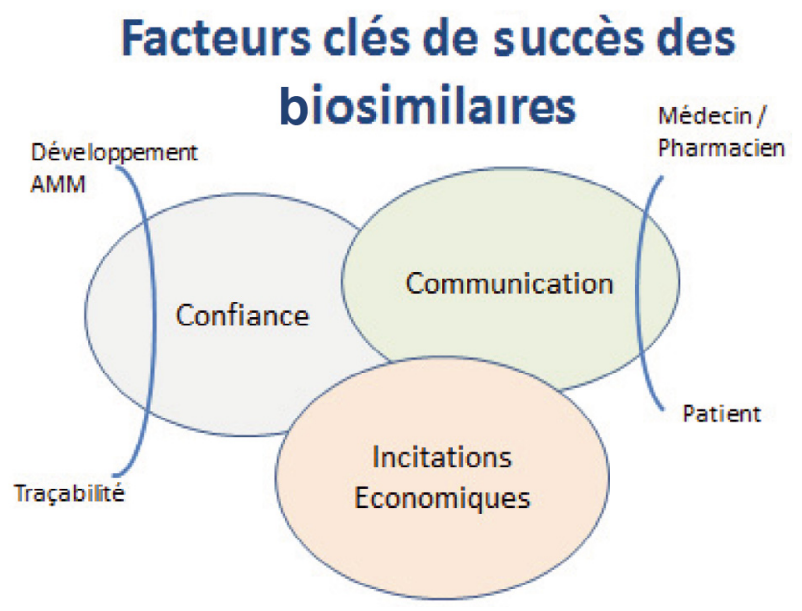

Fig. 1. Facteurs clés de succès des biosimilaires.

AMM : autorisation de mise sur le marché.

Pour soutenir les professionnels de santé dans cette création de confiance, une communication institutionnelle sur les biosimilaires, adaptée aux publics concernés (professionnels de santé, patients), partagée avec les sociétés savantes et les associations de patients et délivrée par tous les acteurs de notre système de soins (ANSM, HAS, Ministère de la Santé, CNAM, Mutualité...), est un élément fondamental.

Les agences de santé ont un rôle majeur dans la mise en place des éléments de cette communication : l'ANSM pour la création de la liste de biomédicaments (molécules princeps et biosimilaires) donnant à tous les professionnels de santé une information exhaustive, et l'HAS pour la réalisation de recommandations médicoéconomiques par classe permettant de sensibiliser les professionnels de santé aux alternatives les plus efficientes et pour la diffusion des fiches de bon usage.

Par ailleurs, une politique incitative au développement des biosimilaires doit tenir compte des circuits de prescription et de délivrance, différents entre ville et hôpital.

Pour les médicaments biosimilaires utilisés dans les établissements de soins, le Comité du Médicament et des Dispositifs Médicaux Stériles (COMEDIMS, ou toute émanation équivalente de la Commission Médicale d'Etablissement [CME]) joue un rôle essentiel dans le choix conjoint cliniciens /pharmaciens des médicaments référencés et inscrits sur le livret thérapeutique.

Parmi les pistes évoquées pour développer les biosimilaires à l'hôpital, citons : l'intégration du biosimilaire dans les contrats de bon usage, les incitations financières au prescripteur ou au service tel qu'un reversement fléché d'une partie des économies réalisées (écart médicament indemnisable [EMI] pour les médicaments de la liste en sus), la réalisation d'essais cliniques ou le développement de l'éducation thérapeutique. Un retour non fléché des économies ou une utilisation uniquement pour combler un 
déficit de l'hôpital seraient certainement moins visibles et moins efficaces.

Pour les médicaments biologiques délivrés en ambulatoire, le médecin prescripteur doit rester mâtre de la décision thérapeutique, le pharmacien relayant les informations auprès des patients. Des incitations positives sous la forme d'objectifs de prescriptions pour les prescripteurs de type résultats sur objectif de santé publique (ROSP) sont à explorer, aussi bien pour les médicaments prescrits en médecine de ville que pour les médicaments prescrits à l'hôpital mais délivrés en ville (PHEV).

\section{Propositions/recommandations de la table ronde}

La table ronde a émis un certain nombre de recommandations pour que le développement du marché des biosimilaires soit un succès, axées sur l'information des acteurs de santé et des patients sur ces médicaments et leurs spécificités, le suivi des traitements aussi bien pour le traitement délivré au patient que pour le suivi de la pharmacovigilance des produits, ainsi que les procédures d'accès au marché.

\section{1. De l'intérêt de disposer d'un registre des médicaments biologiques disponibles sur le marché}

Force est de constater que la notion même de médicament biologique et de leurs spécificités reste actuellement assez confuse pour nombre des acteurs de santé (médecins, pharmaciens de ville et hospitaliers, patients ) : la TR recommande la mise à disposition d'une liste exhaustive des biomédicaments, à laquelle on puisse se référer. Cette liste pourrait être établie par une agence règlementaire, de façon similaire au registre des médicaments actuellement disponible sur le site de l'ANSM.

\subsection{De l'intérêt d'un dossier pharmaceutique (DP) pour tout patient traité par un biomédicament avec une mémoire longue pour couvrir les durées de traitement}

Pour que les modalités d'initiation de traitement par un biosimilaire, recommandées par l'ANSM, puissent être appliquées, tout pharmacien doit pouvoir avoir accès à l'historique de traitement de chaque patient traité par un biomédicament : la TR recommande la mise en place d'un dossier pharmaceutique pour tout patient pour qui est initié un tel traitement, afin que ce dossier soit complété à chaque délivrance de biomédicament, aussi bien en ville qu'à l'hôpital. A l'image de ce qui a été mis en place pour la traçabilité des produits dérivés du sang, le DP est le garant pour disposer de l'historique de traitement des patients, et éviter les éventuelles alternances d'un produit à l'autre (médicament de référence vers biosimilaire, biosimilaires entre eux, biosimilaire vers le médicament de référence). Compte tenu de la durée de traitement de ces biomédicaments, il est nécessaire que la sauvegarde de ce DP soit prolongée dans le temps, et soit étendue par rapport à la sauvegarde actuelle du DP qui n'est que de quelques mois. Sa mise à disposition doit être partagée entre les pharmacies hospitalières et de ville, afin de pouvoir garder trace d'une substitution qui aurait été effectuée à la primo prescription hospitalière et ainsi poursuivre le traitement avec le même produit lors du renouvellement en ville, conformément aux recommandations de l'ANSM. ${ }^{[14]}$

\subsection{De l'intérêt d'un suivi de pharmacovigilance de qualité pour les biosimilaires}

En complément des plans de gestion de risque (PGR), mis en place pour tout biomédicament au moment de l'AMM, il est proposé que tout patient à qui est prescrit un biomédicament (médicament de référence ou biosimilaire) soit inscrit dans un registre prospectif, à l'instar de ce qui est fait dans certains pays (pays nordiques par ex.). Ceci permettrait un suivi longitudinal des patients traités, d'analyser les données de tolérance ainsi que les traitements reçus en vie réelle. L'accord de la Commission Nationale Informatique et Libertés (CNIL) est nécessaire pour la mise en place de ce type de registres, et il est apparu à de nombreux membres de la table ronde que l'inscription à ces registres puisse être une contrepartie de l'accès des patients au remboursement (généralement à $100 \%$ ) de ces traitements onéreux. Ces registres ou études observationnelles, comparables aux études post-AMM mises en place à la demande de la commission de la transparence pour un grand nombre de biomédicaments, contribueront à fournir des données d'efficacité et de sécurité d'emploi en conditions réelles d'utilisation, notamment en comparaison au produit de référence. Ils fourniront les données nécessaires à instaurer la confiance des professionnels de santé et des patients sur l'utilisation des biosimilaires aux côtés de leurs molécules de référence.

\subsection{De l'intérêt d'une procédure simplifiée pour l'accès au remboursement des biosimilaires}

Concernant l'évaluation des conditions de remboursement, il est proposé une procédure accélérée par la Commission de la Transparence, dont les modalités restent à définir, permettant des négociations de prix plus rapides avec le CEPS et de raccourcir les délais d'accès au marché. Des règles stables et transparentes devront être élaborées par le CEPS dès lors que la procédure d'évaluation qui précède la fixation du prix sera stabilisée. 
7.5. De l'intérêt d'une communication envers les médecins et pharmaciens, ainsi que les patients, en complément des mesures d'incitations pour les biosimilaires

Le succès de la progression des biosimilaires dans les pratiques de soins ne peut se concevoir sans transparence et communication préalable avec les acteurs de santé.

Quelles que soient les mesures d'incitation à la prescription des biosimilaires, et sans minimiser leur impact, le succès d'une politique favorisant la prescription des biosimilaires doit s'appuyer sur la communication : il est essentiel d'instaurer la confiance aussi bien auprès du médecin et du pharmacien que du patient, notamment dans le contexte de pathologies sévères telle que la cancérologie ou certaines pathologies auto-immunes où les choix thérapeutiques sont limités. Le biosimilaire ne peut être comparé à un produit générique, et la pratique de substitution automatique par le pharmacien comme pour un générique n'est aujourd'hui ni recommandée, ni envisageable. L'expérience récente en France sur les génériques montre que la communication doit être repensée pour instaurer la confiance et impliquer les médecins prescripteurs à introduire progressivement les BS dans leur choix de prescription. Seule une communication adaptée peut contribuer à faire reconnaître le biosimilaire comme une alternative à moindre coût à un biomédicament de référence. Il est proposé que des éléments de communication didactiques soient préparés à destination des patients, des prescripteurs et des pharmaciens. Ces documents seront idéalement élaborés en partenariat avec les sociétés savantes et avec les associations de patients, lorsqu'elles existent, afin de répondre au mieux aux attentes de chacun. Il est indispensable que le médecin prescripteur dispose de toutes les informations sur le biosimilaire, afin d'être à même de relayer la communication auprès de son patient, et qu'il soit informé du médicament qui sera effectivement délivré au patient, notamment en cas de primo substitution par le pharmacien, ne serait-ce que pour renouveler la prescription avec le même produit, ainsi que participer pleinement à ses obligations de déclaration de pharmacovigilance, en particulier de notification spontanée.

\section{Conclusions}

L'introduction progressive et réussie des biosimilaires dans l'offre de soins doit s'appuyer sur 3 facteurs clés de succès : la confiance qui se construit grâce à un dossier d'AMM solide avec des preuves d'équivalence étayées par des données de qualité, sécurité et efficacité, et grâce à un système de vigilance et de traçabilité post-autorisation, permettant un suivi de leur tolérance à long terme et une évaluation de l'impact des «switchs »; une communication cohérente auprès des professionnels de santé et des patients associant pouvoirs publics, industriels et sociétés savantes; des incitations économiques centrées sur le médecin dans un premier temps, qui sera le meilleur vecteur de confiance auprès du patient. Ce trépied est indissociable, la confiance ne s'instaurera qu'en respectant toutes ces étapes et en impliquant tous les acteurs du médecin prescripteur au pharmacien et au patient. A terme, les données scientifiques collectées permettront de préciser si les mesures de précaution actuelles recommandant la non substitution automatique et la non interchangeabilité entre les produits de référence et les biosimilaires doivent être maintenues ou peuvent être levées.

Remerciements. Nous remercions vivement l'ensemble des participants à la table ronde $\mathrm{N}^{\circ} 3$, ainsi que le Pr Jacques-Eric Gottenberg (CHU de Strasbourg), Mme Marie-Claude Laubignat (Laboratoire Teva), Mr Frédéric Rupprecht (Sanofi) et le Pr Hervé Le Louet (CHU Mondor, AP-HP ) qui, bien que n'ayant pas pu y participer, ont contribué à la préparation des thèmes abordés lors de cette TR.

Conflits d'intérêt. Danièle Girault, Corine Blachier, François Gary, Didier Laloye, Rima De Sahb Berkovitch, Cécile Rey Coquais sont salariés de l'industrie pharmaceutique (médicaments innovants et/ou biosimilaires); Catherine Lassale est salariée du syndicat de l'industrie pharmaceutique, le LEEM.

Les autres auteurs n'ont pas de conflits d'intérêt à déclarer en rapport avec cet article.

Abréviations. AMM : autorisation de mise sur le marché; ANSM : Agence Nationale de Sécurité du Médicament et des Produits de Santé ; ASMR : amélioration du service médical rendu; BS : biosimilaire; CA : chiffre d'affaire; CEPS : Comité Economique des Produits de Santé; CME : Commission Médicale d'Etablissement; CNAMTS : Caisse Nationale d'Assurance Maladie des Travailleurs Salariés; CNIL : Commission Nationale Informatique et Libertés; COMEDIMS : Comité du Médicament et des Dispositifs Médicaux Stériles ; CSIS : Conseil Stratégique des Industries de Santé ; DP : dossier pharmaceutique; EGA : European Generic Association; EMA : Agence Européenne du Médicament; EMI : écart médicament indemnisable; EPO : érythropoiétine; GCSF : granulocyte colony-stimulating factor; HAS : Haute Autorité de Santé ; HBPM : héparine de bas poids moléculaire; LEEM : Les Entreprises du Médicament; LFSS : loi de financement de la Sécurité Sociale; $\mathrm{M} €$ : million d'euros; $\mathrm{Md} €$ : milliard d'euros; PGR : plan de gestion du risque; PHEV : prescriptions hospitalières exécutées en ville; PIB : produit intérieur brut; SMR : service médical rendu; TR : table ronde.

\section{Références}

1. Guideline on similar biological medicinal products (CHMP/437/04 Rev 1) http: //www . ema. europa.eu Consulté le 26 décembre 2014 
2. Directive $2001 / 83 / \mathrm{EC}$ on the Community code relating to medicinal products for human use

http: //www . europa.eu

Consulté le 26 décembre 2014

3. Questions et réponses relatives aux médicaments biosimilaires (médicaments biologiques similaires). 2011

http://www.ema.europa.eu/docs/fr_FR/document_library/ Medicine_QA/2009/12/WC500020062.pdf

Consulté le 26 décembre 2014 (2 pages)

4. Trouvin J.-H. Caractéristiques des biosimilaire. In Prugnaud J.-L. Trouvin J.-H. Les biosimilaires (chaptire 1). Springer Paris 2001; 1-27

5. Guideline on similar biological medicinal products containing biotechnologyderived proteins as active substance: quality issues (revision 1). EMA/CHMP/BWP/247713/2012. 2012

http://www . europa . eu

Consulté le 26 décembre 2014

6. Guideline on similar biological medicinal products containing biotechnologyderived proteins as active substances: non-clinical and clinical issues. EMEA/CHMP/BMWP/42832/2005. Guideline currently under revision. 2005

http://www . europa.eu

Consulté le 26 décembre 2014

7. Assessment report, Remsima.

http: //www.ema.europa.eu/docs/en_GB/document_library/ EPAR-public_assessment_report/human/002576/WC500151486. $\operatorname{pdf}$

http: //www. ema. europa.eu

Consulté le 26 décembre 2014

8. Weise M, Kurki p, Wolff-Holz E, et al. Biosimilars: the science of extrapolation. Blood 2014 doi:10.1182/blood-2014-06-583617

9. http://www.imshealth. com

Consulté le 26 décembre 2014
10. Comité Economique des Produits de Santé. Rapport d'activité 2013 http://www . sante.gouv.fr/les-activites-du-ceps.html Consulté le 26 décembre 2014

11. Accord cadre du 5 décembre 2012 entre le CEPS et les entreprises du médicament. http://www. sante.gouv.fr/IMG/pdf/accord_cadre_ du_051212.pdf Consulté le 26 décembre 2014 (24 pages)

12. 6 è édition du Conseil Stratégique des Industries de Santé 5 juillet 2013 http://www. sante.gouv. fr/un-plan-operationnel-pour-lesindustries-et-technologies-de-sante-au-service-de-lasante-de-1-innovation-de-1-emploi-et-de-la-croissance. html Consulté le 26 décembre 2014

13. Loi de financement de la Sécurité Sociale. 2014 article 47 http://www . legifrance.gouv.fr/affichTexte. do?cidTexte=JORFTEXTOQ0028372809\&fastPos $=1 \&$ fastReqId $=1017469037 \&$ categorieLien=cid \& oldAction $=$ rechTexte Consulté le 26 décembre 2014

14. Rapport ANSM. Les médicaments biosimilaires : états des lieux. Septembre 2013

http: //ansm. sante.fr/S-informer/Points-d-informationPoints-d-information/L-ANSM-publie-un-etat-des-lieuxsur-les-medicaments-biosimilaires-Point-d-information Consulté le 26 décembre 2014

Correspondance et offprints : Danièle Girault, Laboratoire Novartis Pharma, 2-4 rue Lionel Terray, BP 308, 92506 Rueil Malmaison, France

E-mail : daniele.girault@novartis.com 